Professor Mihaela MUNTEAN, PhD

E-mail: mihaela.muntean@e-uvt.ro

Department of Business Information Systems

West University of Timisoara

Associate Professor Claudiu BRÂNDAŞ, PhD

E-mail: claudiu.brandas@e-uvt.ro

Department of Business Information Systems

West University of Timisoara

Associate Professor Marian Pompiliu CRISTESCU, PhD

E-mail: marian.cristescu@ulbsibiu.ro

Department of Finance and Accounting

"Lucian Blaga" University of Sibiu

Diana MATIU

E-mail: matiu_diana@yahoo.com

SAP Romania

\title{
IMPROVING CLOUD INTEGRATION USING DESIGN SCIENCE RESEARCH
}

Abstract. Cloud integration offers new opportunities to streamline the business processes in organizations. Variate integration approaches are possible: Cloud-to-Cloud, Cloud-Integrator-Cloud or Cloud-Cloud-Cloud. Depending on the cloud service, we identify various cloud service models, namely Anything as a Service (XaaS). We propose an activity framework for deploying a CloudIntegrator-Cloud (CIC) integration of two Software as a Service (SaaS) systems. The demarche has an achievement in the implementation of two SAP Cloud software. The software integration is subject of a SCRUM process management approach. We consider the integration framework and the integration process implementation artefacts, outputs of a software project developed in terms of the Design Science Research (DSR) process. Based on relevant literature references on DSR, we apply DSR principles to the CIC integration problem. The DSR process steps are mapped to the SCRUM sprints introduced in the integration project. Model.

Keywords: Design Science Research, Software Integration, Cloud Service

JEL Classification: L20, L21, L86, M15

1. Introduction

Design Science Research (DSR), according to the reference syllabi „Design Research in Information Systems” (see Van der Merwe) addresses two key issues: „the central role of the information system/technology artifact

DOI: 10.24818/18423264/55.1.21.13 
and the importance of professional relevance of information system research". Information system design is performed with respect to the business stategy and the global organizational design (Figure 1).

March and Smith (1995) identify two design processes and four design artifacts produced by Design Science Research. Whether or not the artifact is perpetuated depends on the way it is designed, on the interface with the external environment and the adaptation of one with the other, given that the implementation of the artifact within organizations causes disruptions in the business environment (see Leoz, 2018).

The artefacts can be models, frameworks, methods (see Hevner, 2004). Guidelines for design science in information systems proposed in Hevner's research are: design as an artefact; problem relevance; design evaluation; research contribution; research rigor; design as a search process; communication of research.

According to Vaishnavi, Kuechler and Petter (2019) „Design Science Research is a set of synthetic and analytical techniques performing research in information systems". It involves the creation of an artefact, e.g. algorithm, human/computer interface, and system design methodology or language. Any DSR approach is a process containing the following phases (Figure 2) (see Takeda, 1990).

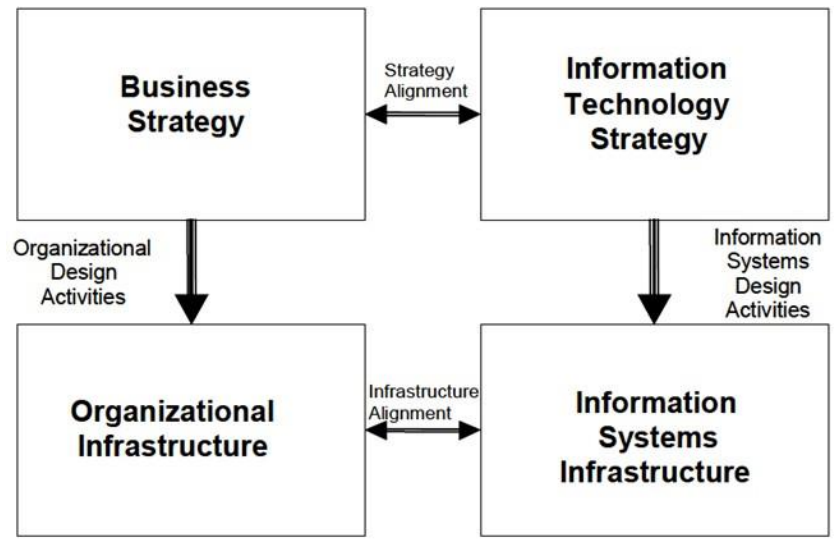

Figure 1. Organizational Design and Information System Design (Hevner 2004)

The conclusion, in form of a knowledge contribution, brings improvements in solving the problem (Beck, 2013).

In the paper "Understanding Design Research. Toward an Achievement of Balance" Owen states "knowledge is generated and accumulated through action". The general model involves undertaking something and evaluating the results obtained. According to this model, the 
process is presented as "a cycle in which knowledge is used, creatively, to obtain things, they are evaluated and thus knowledge is obtained".

An innovative proposal, e.g. model, framework, architecture, methods, design principle or theory, improves the effectiveness and efficiency of the information systems/technology adopted by the business environment.

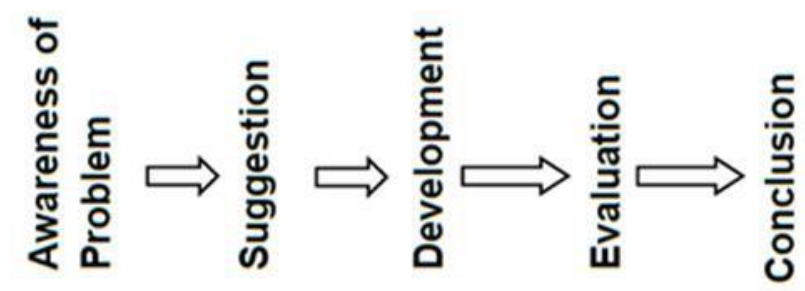

Figure 2. DSR process (Vaishnavi, 2019)

Information system, viewed as an artifact, reflects the relationships between its components (modules, interfaces, frameworks), which makes some behaviors to be supported while others are subject to constraints.

Models are sets of propositions or statements expressing relationships between constructs. Frameworks establishes real or conceptual guides to serve as support or guide. In this paper, we introduce a framework for Cloud-Integrator-Cloud (CIC) integration. Implementation aspects make referees to SAP Hybris Commerce and SAP Cloud for Customers (SAP C4C). The study case, developed as a DSR process, follows the transfer of an insurance policy from SAP Hybris Commerce to SAP C4C.

Architectures are high-level structures of systems. The proposed integration framework and the concrete implementation can determinate the architecture of a Software as a Service (SaaS) solution for integrating SAP Hybris Commerce with SAP Cloud for Customer.

In accordance with DSR principles, the process of designing, realizing and implementing information systems (IS) can be linked to the methods and techniques used in architecture and construction. Thus, an IS must be:

- functional - with a high level of reliability, without errors and defects that prevent the users to use their capabilities;

- useful - it must be developed based on the users' requirements and perfectly adapted to them;

- effective - to increase user satisfaction and give them a pleasant experience in the actual use process.

Further, design principles are core principles and concepts to guide design, while design theories are prescriptive set of statements on how to do something to achieve a certain objective (Vaishnavi, 2019).

DOI: 10.24818/18423264/55.1.21.13 


\section{Theoretical and practical perspective of cloud integration \\ 2.1 Cloud service models}

Cloud integration implies bringing together applications and systems in a unitary cloud framework. Data and cloud services are available to anyone who has access to the integrated system. The as-a- service ("aaS") model, in the context of cloud computing, is a term that indicates a service, which is available over the Internet via the cloud (Doug, 2019). Based on the service the cloud model is offering we identify various cloud service models, namely Anything as a Service (XaaS).

Infrastructure as a Service (IaaS), Platform as a Service (PaaS) and Software as a Service (SaaS) are the three main categories of cloud computing (Figure 3). IaaS offers access to high performant computers, networks and storage devices as needed. PaaS provides a platform for software development and offers a variety of services for development, testing and deployment. SaaS is a cloud application service and utilize the internet to deliver applications to its users (Palos-Sanches, 2017; Chody, 2018).

Cloud-computing adoption by organizations increases rapidly due to ascertained advantages (Columbus, 2018; Gil, 2019). It implies product/service innovations, process innovations, organizational innovations, and technological innovations. Cross-functional processes that are spread out across several applications need the integration of many XaaS services. Data managed by a specific XaaS services, e.g. SaaS service, is required in another $\mathrm{XaaS} / \mathrm{SaaS}$ services in order to optimize the business processes.

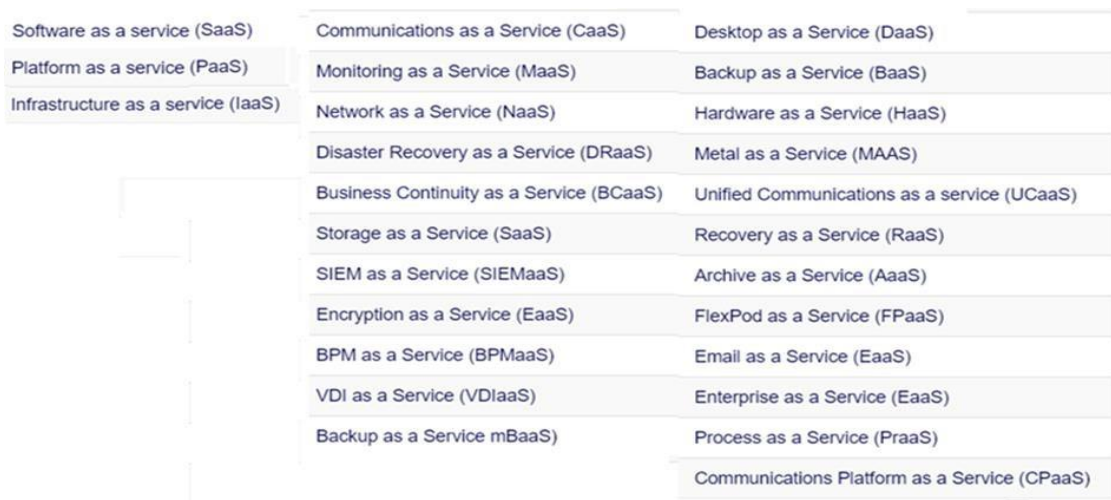

Figure 3. Cloud service models. Anything as a Service (source: https://www.orosk.com/cloud- computing/) 
For supporting Business-to-Business (B2B) and Business-to-Consumer (B2C) processes, we call for variate integration approaches: Cloud-to-Cloud, Cloud-Integrator-Cloud or Cloud-Cloud-Cloud (Cloud-Cubed) (Wall Street Journal, 2013).

\section{CLOUD-TO-CLOUD INTEGRATION}

As companies embrace more SaaS applications, the need to integrate the SaaS applications will increase. Various integration techniques use web services (Table 1) to implement the integration process (Linthicum, 1999; Kolluru, 2013). Web services are Extensible Markup Language (XML) - centered data exchange systems that use the Internet for Application-to-Application (A2A) communication and integration (Wang, 2014), (Linthicum 1999), (Muntean, 2019). A comprehensive overview upon Simple Object Access Protocol (SOAP), REST (Representational State Transfer) and ODATA (Open Data Protocol) services reflect the differences between them (Muntean, 2019).

Table 1. Web services overview (Linthicum, 1999)

\begin{tabular}{|l|l|}
\hline Web service & \multicolumn{1}{|c|}{ Characteristics } \\
\hline SOAP & $\begin{array}{l}\text { A service architecture; XML based; Runs on HTTP but envelopes } \\
\text { the message; } \\
\text { Slower than REST; Very mature, a lot of functionality; Not suitable } \\
\text { for browser-based clients }\end{array}$ \\
\hline REST & $\begin{array}{l}\text { A service architecture (resource-oriented); Uses the HTTP } \\
\text { headers to hold meta-information (although it is protocol- } \\
\text { agnostic); Can be used with XML, JSON or whatever necessary; } \\
\text { Usually used with JSON due to the easily parsable content; Faster } \\
\text { than SOAP; It uses semantic media types }\end{array}$ \\
\hline OData & Protocol; Based on HTTP, AtomPub and JSON; RESTful by design \\
\hline
\end{tabular}

An important issue in the cloud environment is to integrate and ensure the quality of transferred and processed data (Muntean, 2019). The integration framework based on the OData service proposed by Muntean and her colleagues in 2019 conducted to a Cloud-to-Cloud integration of two SAP cloud software, SAP Cloud for Customer and S/4 HANA Cloud.

\section{CLOUD-INTEGRATOR-CLOUD INTEGRATION}

The integration platform (Figure 4) receives data from one cloud/SaaS application, convert it into the format required by the receiver

DOI: $10.24818 / 18423264 / 55.1 .21 .13$ 
cloud/SaaS application, and send the data by invoking the web services exposed by the receiving application (Wall Street Journal, 2013).

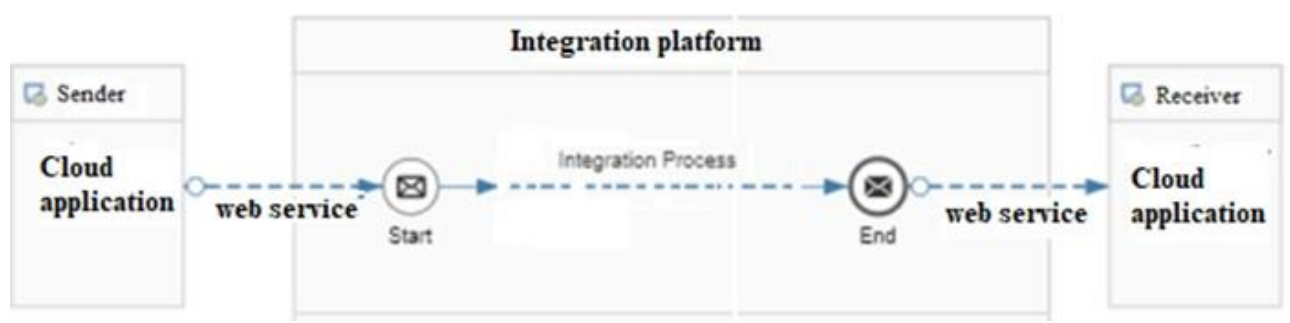

Figure 4. Cloud-Integrator-Cloud integration

Due to the extended integration capabilities, the platform integrates data and processes in Application-to-Application (A2A) and Business-to-Business scenarios. SAP Cloud Platform Integration (SAP CPI) is representative for our demarche. It is a cloud-based integration platform for integrating cloud applications with other SAP and non-SAP cloud and on premise solutions. SAP CPI supports SOAP over HTTP and HTTPS protocols. The connectivity adapters allow the implementation of the integration process via web services, e.g. OData service (SAP Help Portal).

\section{CLOUD-CLOUD-CLOUD INTEGRATION}

This type of integration is similar to the cloud-integrator-cloud approach, except that the integration system is also a cloud-based application, e.g. Oracle Integration Cloud Service or others Integration Platform as Service (iPaaS) solutions.

\subsection{Cloud-Integrator-Cloud integration}

The integration platform sustains the integration process, all the necessary tasks to transfer the data send to the receiver application (Figure 5).

Activity A4 defines the main integration flow. Additionally, security aspects are settled in A5 and error handling is introduced in A6. Finally, the data is copied into the receiver application according to the previously defined senderreceiver mapping (activity $\mathrm{A} 7$ ).

Digital businesses are highly integrated and innovative. Integration platforms are commonly used for integrating different applications and services. In a Cloud-Integrator-Cloud (CIC) integration framework, the integrator receives the data from one cloud application, convert it into the format required by the other application and send it by invoking web services (Mittal, 2017). 
Improving Cloud Integration Using Design Science Research

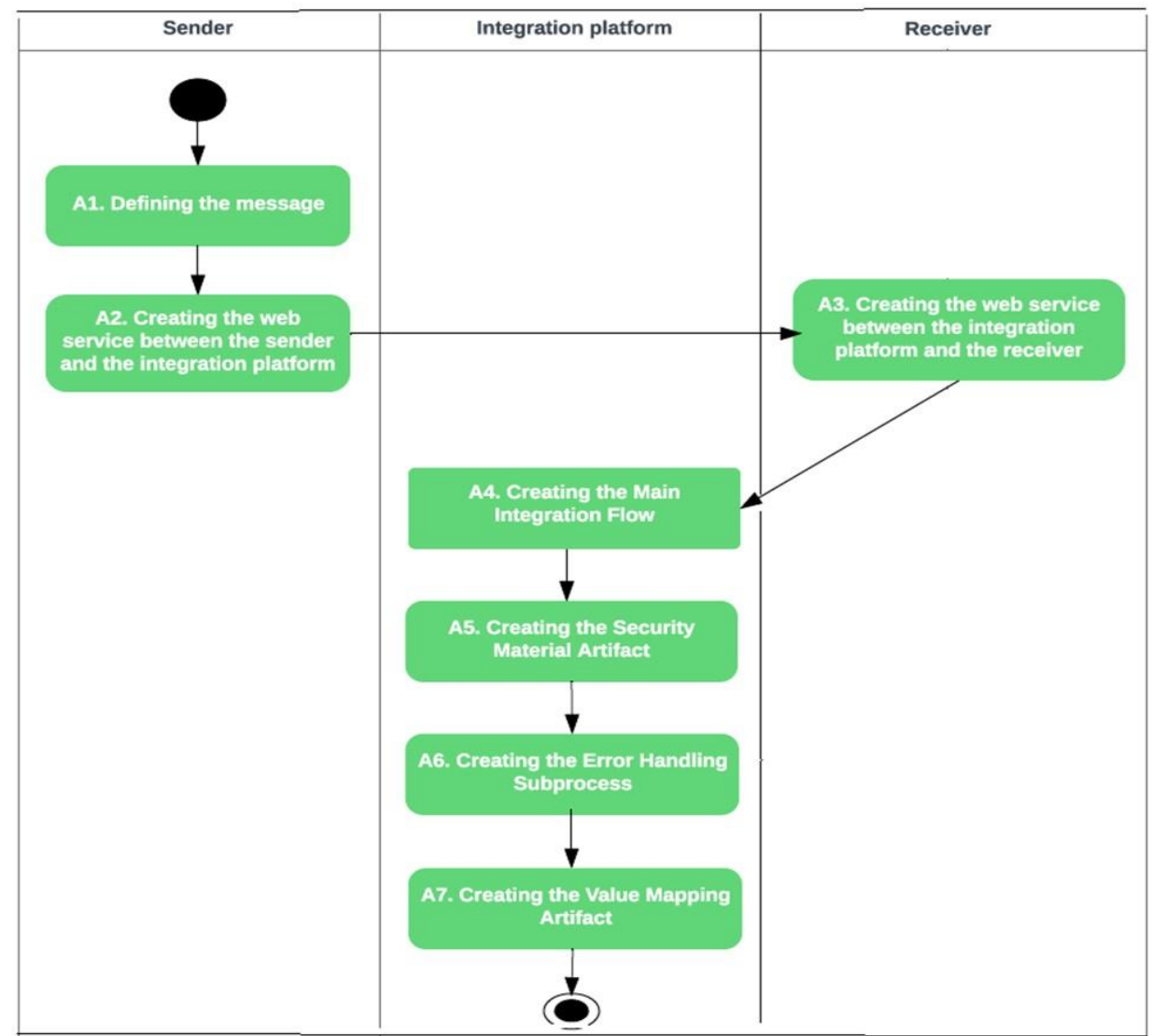

Figure 5. Framework for Cloud-Integrator-Cloud integration (CIC integration)

\subsection{CIC integration in the cloud environment}

SAP Cloud Platform Integration (SAP CPI) suite allows the integration of processes, data and devices by using different methods for consuming cloud services, open Application Programming Interfaces (APIs) and tools (Techtarget,2018), (Vaishnavi,2004).

There can be a variety of business use cases (Sharma, 2018), but all of them start from the business and customer perspective: replication of customer profile from SAP Hybris Commerce to SAP C4C; directly access of a customer from SAP Hybris Commerce to SAP C4C in order to create a service ticket; directly access from SAP C4C to SAP Hybris Commerce and deliver support to the customer (Pillai, 2015).

Integration projects in the cloud imply various agile approaches, like Crystal methodologies, Extreme Programming, Feature-Driven Programming,

DOI: 10.24818/18423264/55.1.21.13 
Mihaela Muntean, Claudiu Brândaş, Marian Pompiliu Cristescu, Diana Matiu

Kanban and SCRUM (Hart, 2010).

An agile approach in the SAP environment implies the following steps (Hevner,2015): 1 - to understand what agile means and what the chosen methodology, e.g. SCRUM; 2 - to identify the stakeholders, e.g. exec management, business owners, development and testing teams, for implying them into the project; 3 - to build a business case, e.g. for a CIC integration with SAP CPI; 4 - to "start small" meaning to start with one team; 5 - to reorganize teams and establish how they are structured and communicate; in SAP projects usually work many small, multifunctional teams; in SCRUM approaches a business representative is the product owner/representative; 6 - to create a prioritized backlog; 7 - to organize the sprint meetings; 8 - to choose some tools to support the agile development process, e.g. Jira, Rally.

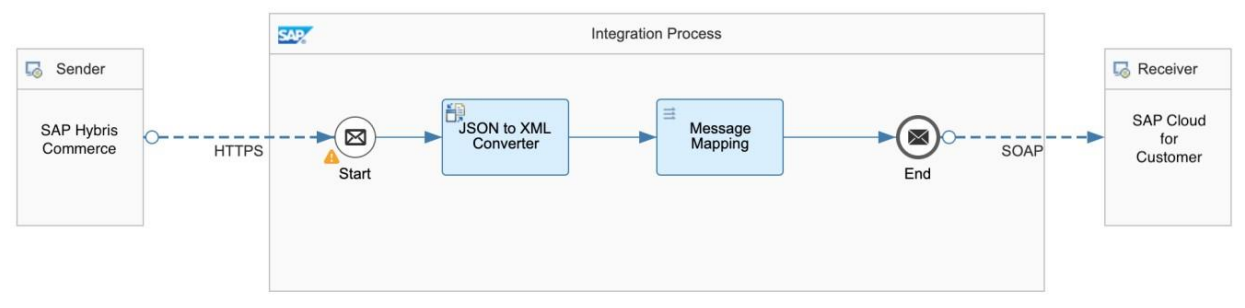

Figure 6. CIC integration with SAP CPI (SAP, 2019)

Based on the integration framework introduced in paragraph II B (Figure 5), the backlog for implementing the considered CIC integration is the following (Figure 7).

Activities A1-A7, strictly related to technology, imply directly the sender, receiver and the integration platform. Additionally, two activities, A0 and A8, complete the development approach (Figure 7).

The deployment of A0 takes into consideration the IT players, but is not executed by neither the sender, receiver nor the integration platform. Activity A8 takes place independently from SAP CPI, SAP Hybris Commerce and SAP Cloud for Customer.

According to SCRUM methodology, the nine activities have story points assigned directly proportional to their complexity. Analyzing the overall project, we propose five sprints for deploying the integration of SAP Hybris Commerce with SAP C4C. 
Improving Cloud Integration Using Design Science Research

\begin{tabular}{|c|c|c|c|c|c|}
\hline $\begin{array}{c}\text { Sprint } \\
\text { No }\end{array}$ & $\begin{array}{c}\text { Activity } \\
\text { ID }\end{array}$ & Activity & $\begin{array}{c}\text { Task } \\
\text { ID }\end{array}$ & Task & $\begin{array}{l}\text { Story } \\
\text { Points } \\
\end{array}$ \\
\hline $\mathbf{0}$ & A0 & \multicolumn{3}{|c|}{ Awareness of the Problem and Tentative Design } & 15 \\
\hline \multirow[t]{6}{*}{1} & \multirow[t]{3}{*}{$\mathbf{A 1}$} & Defining the message & T11 & Sprint Planning & \multirow[t]{3}{*}{13} \\
\hline & & & $\mathrm{T} 12$ & Defining the body of the message & \\
\hline & & & $\mathrm{T} 13$ & Defining the headers of the message & \\
\hline & \multirow[t]{3}{*}{$\mathbf{A 2}$} & $\begin{array}{l}\text { Creating the web service between } \\
\text { SAP Hybris Commerce and SAP CPI }\end{array}$ & $\mathrm{T} 21$ & Defining the operation of the web service & \multirow[t]{3}{*}{7} \\
\hline & & & $\mathrm{T} 22$ & Configuring the authentication method in SAP CPI & \\
\hline & & & $\mathrm{T} 23$ & Configuring the integration flow endpoint & \\
\hline \multirow[t]{5}{*}{2} & \multirow[t]{5}{*}{$\mathbf{A} 3$} & $\begin{array}{l}\text { Creating the web service between } \\
\text { SAP CPI and SAP C4C } \\
\end{array}$ & $\mathrm{T} 31$ & Creating the Communication System & \multirow[t]{5}{*}{15} \\
\hline & & & $\mathrm{T} 32$ & Configuring the Communication Scenario & \\
\hline & & & T33 & Creating and configuring the Communication Arrangement & \\
\hline & & & T34 & Configuring the authentication method in SAP C4C & \\
\hline & & & $\mathrm{T} 35$ & Defining the endpoint & \\
\hline \multirow[t]{8}{*}{3} & \multirow[t]{8}{*}{$\mathbf{A 4}$} & Creating the Main Integration Flow & $\mathrm{T} 41$ & Defining and configuring the Sender System & \multirow[t]{8}{*}{20} \\
\hline & & & $\mathrm{T} 42$ & Configuring the Adapter of the Sender System & \\
\hline & & & $\mathrm{T} 43$ & Configuring the Converter & \\
\hline & & & T44 & Defining the Content Modifier for the message headers & \\
\hline & & & $\mathrm{T} 45$ & Configuring the Routers of the message & \\
\hline & & & $\mathrm{T} 46$ & Creating the Message Mappings & \\
\hline & & & T47 & Defining and configuring the Receiver System & \\
\hline & & & $\mathrm{T} 48$ & Configuring the Adapter of the Receiver System & \\
\hline \multirow[t]{6}{*}{4} & A5 & \multicolumn{3}{|l|}{ Creating the Security Material Artifact } & 3 \\
\hline & \multirow[t]{3}{*}{ A6 } & $\begin{array}{l}\text { Creating the Error Handling } \\
\text { Subprocess }\end{array}$ & T61 & Defining the Content Modifier for message headers & \multirow[t]{3}{*}{8} \\
\hline & & & T62 & Catching the error message & \\
\hline & & & T63 & Converting and modifying the error message & \\
\hline & A7 & \multicolumn{3}{|l|}{ Creating the Value Mapping Artifact } & 4 \\
\hline & $\mathbf{A 8}$ & Disseminate the results & & & 5 \\
\hline
\end{tabular}

Figure 7. CIC integration. Backlog

\section{Research strategy. Applying the DSR process to CIC integration}

The scientific approach of the methods and techniques of research design in the field of IS, implies the division of the research effort in two directions: on the one hand, the focus is on the set of activities that configure a process, e.g. the cloud integration process, and on the other hand the focus should be on the product, viewed as an artifact, a result of the process, e.g. the integration scenario. The DSR process uses past knowledge in its running and can contribute to enrich the knowledge base in a given area. The proposed integration scenario, its development and implementation represents a best practice artefact that can be further used for similar projects in the application domain (Figure 8).

Due to the increased use of SAP cloud solutions and the necessity to integrate these systems in order to support business processes that span multiple organizational areas, the research was oriented on SAP cloud integration application domain.

After investigating the application domain from theoretical and practical point of view and evaluating some ongoing integration projects, we have considered SAP Hybris Commerce and SAP Cloud Customer the pillars of our integration framework.

DOI: 10.24818/18423264/55.1.21.13 
Mihaela Muntean, Claudiu Brândaş, Marian Pompiliu Cristescu, Diana Matiu

According to the DSR process, the integration process was modeled, each stage/step is further explicitly outlined in the presentation of the research.

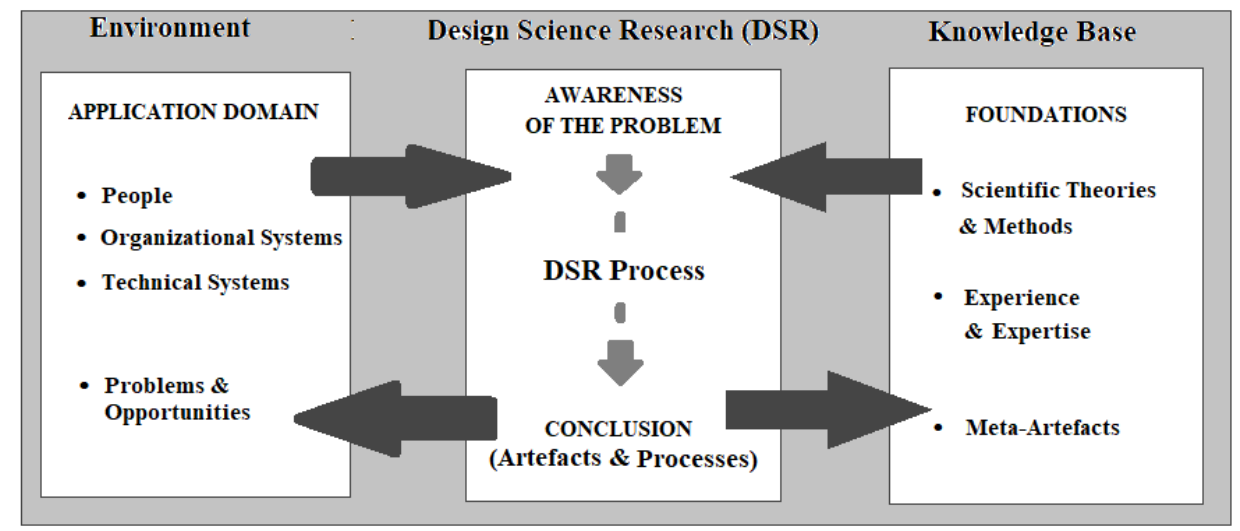

Figure 8. The DSR process and its connectivity with the environment and the knowledge base (Kapinga, Montero, Mbise, 2019)

\section{AWARENESS OF THE PROBLEM}

In our paper, the starting point, in this stage, is the detailed analysis of the two systems (SAP Hybris Commerce and SAP C4C), which are the object of the integration process, and the integration platform (SAP Cloud Platform Integration).

The CIC integration of the two systems implies the transfer of an insurance policy from SAP Hybris Commerce to SAP C4C. The integration process is part of the following business process:

(1) Step 1: A business consultant fills in a form with the client personal data.

The client assist the consultant during this step.

(2) Step 2: The insurance policy, containing the client data, is generated.

(3) Step 3: The client gives his consent and signs the insurance policy.

(4) Step 4: The consultant activates the policy generation button and the data is automatically replicated into SAP Cloud for Customer software.

(5) Step 5: SAP Cloud for Customer maintains the client data and the insurance policy in pdf format.

\section{SUGGESTION}

The suggestion is a creative stage in which the aim is to obtain a new functionality, of the integration framework, based on a new or existing configuration in which new or existing applications and services appear. The objective of this phase consists in a proposal for the integration process in order to support the business process (Figure 9). 


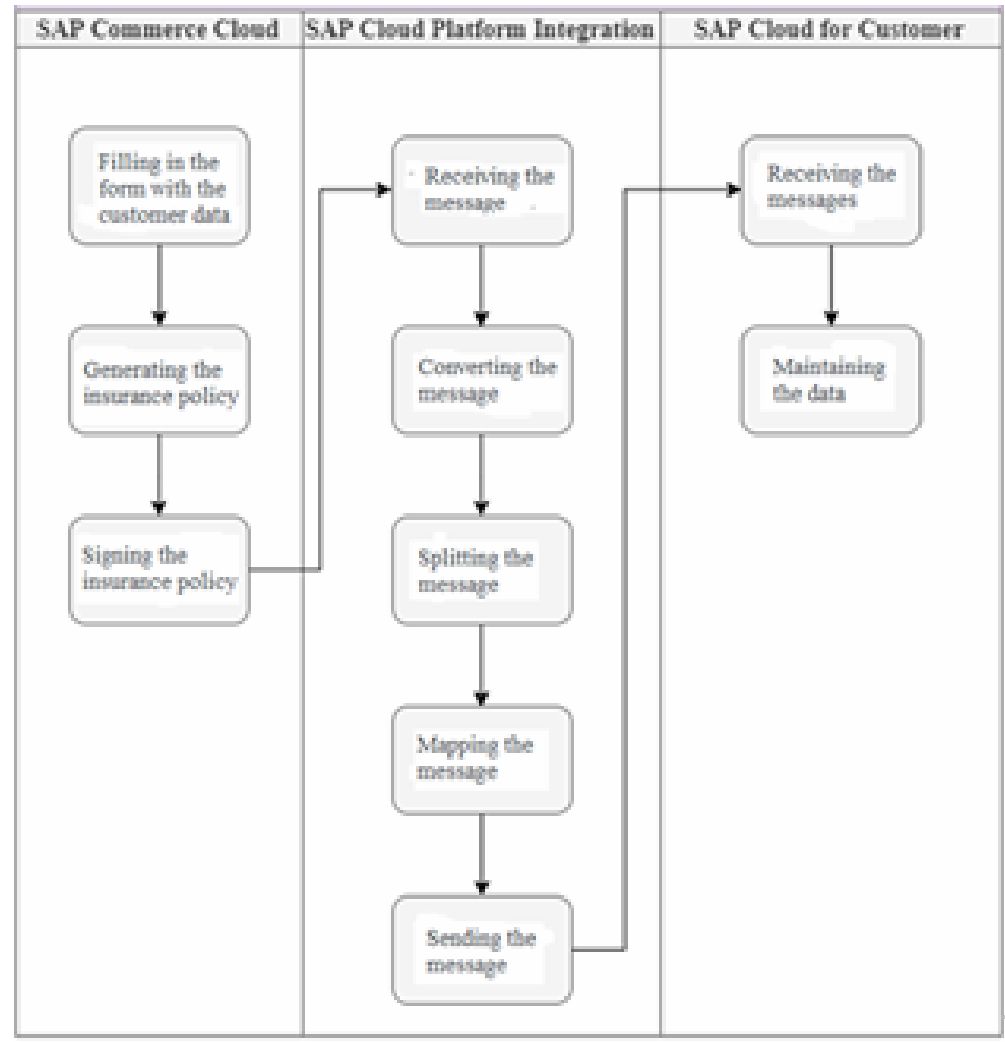

Figure 9. Integration scenario

SAP CPI represents the suggested integration alternative, despite a directly Cloudto-Cloud variant.

\section{DEVELOPMENT}

The constructions and the specific approach of the CIC reveal the vocabulary and symbols necessary to define the specific SAP problems and to find the appropriate solutions to the integration process. All these aspects have a major impact at this stage, especially on the way the tasks are designed and the problems to be solved are dealt with by the members of the integration team.

The tentative design of the integration process (Figure 9) is further developed according to the general CIC framework and the project backlog is established (Figure 10). After establishing all activities (A1 - A7) and the individual tasks (T11 - T63), sprint planning is necessary.

This means to define the deliveries for each sprint and how that is achieved. Sprint planning implies the collaboration with the whole SCRUM team (Figure 10).

DOI: $10.24818 / 18423264 / 55.1 .21 .13$ 
Mihaela Muntean, Claudiu Brândaş, Marian Pompiliu Cristescu, Diana Matiu

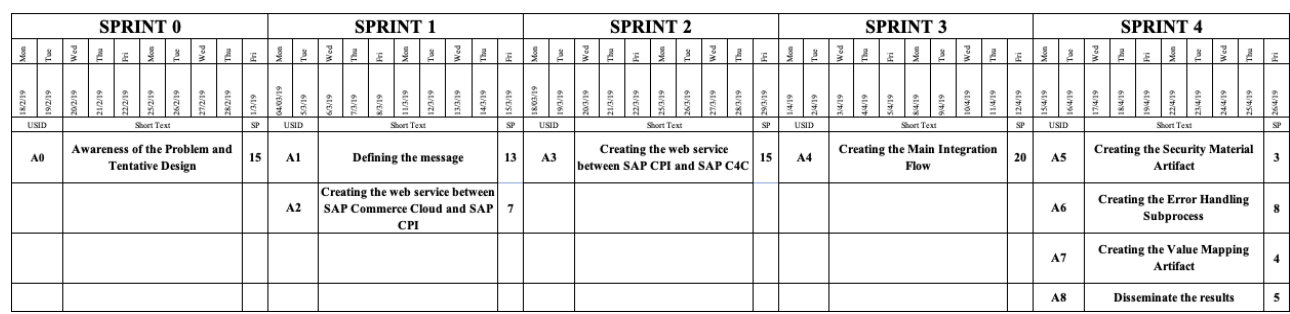

Figure 10. Sprint planning

Finally, the whole integration process is deployed based on the technical capabilities of SAP CPI and of the two involved software (Figure 11).

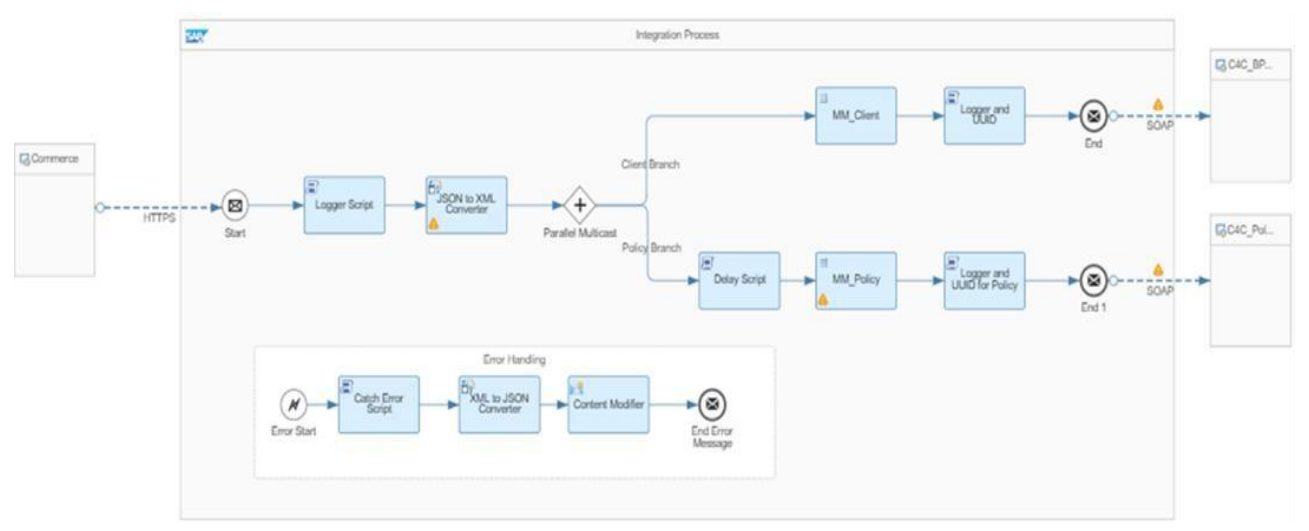

Figure 11. Development. Implementation of the integration process

The message contains the client data, assurance policy data and a pdf attachment with the policy. In the receiver, the data is stored in the client table, policy table and in the attachments table. Therefore, two communications scenario establish the transfer: one for the client data and another for the policy data and the .pdf file.

\section{EVALUATION}

The basic characteristic of this stage, according to the basic principles of DSR is the critical approach of the activities undertaken. Sprints are evaluated, as IT artifacts, in the business environment, in an organization, within which the requirements and criteria based on which they will be evaluated are established. The basic characteristic of this stage, according to the basic principles of DSR is the critical approach of the activities undertaken. Sprints are evaluated, as IT artifacts, in the business environment, in an organization, within which the requirements and criteria based on which they will be

DOI: $10.24818 / 18423264 / 55.1 .21 .13$ 
evaluated are established.

This stage results, in many cases, in identifying the weaknesses of the sprint (viewed as an artifact) and implicitly improving it through refining and reevaluation processes. The sprint evaluation process involves the prior definition of the corresponding values and the collection and analysis of the appropriate data from the development stage. Sprints can be evaluated in terms of functionality, completeness, coherence, accuracy, performance, reliability, usefulness, how they fit with the organization where the development and implementation process takes place, to which other features can be added.

In this process, the aim is to accumulate the necessary and sufficient knowledge regarding the different evaluation techniques, how the questions are asked and their correctness, the method of choosing the most suitable technique, which will give confidence in the results obtained from the evaluation, as a proof of the quality and performance obtained in the sprint design process.

According to SCRUM methodology, each sprint is an iteratively miniproject. Beyond the final evaluation, a proper passed evaluation of each sprint output determinates the moving to the next sprint.

The final test, after sprint 4, implies the sending of different messages, including wrong messages for testing the error handling process. Evaluation means to compare the message (data) received by SAP C4C with the message (data) send by SAP Hybris Commerce.

SAP CPI allows you to view the message details, like properties, content, header, format and message pass (Figure 12).

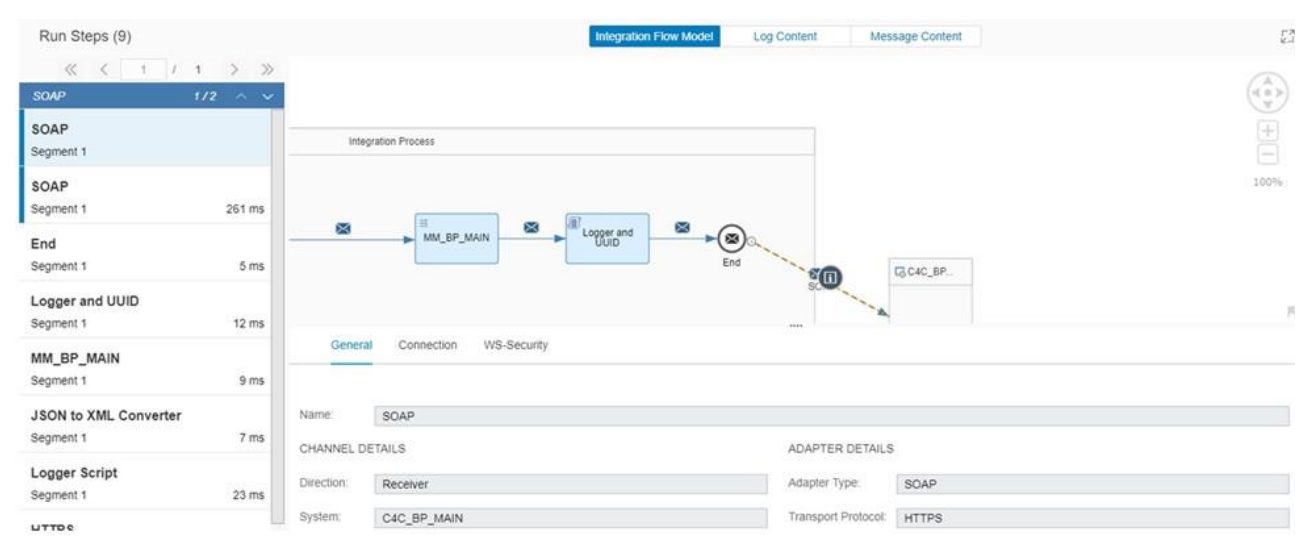

Figure 12. Evaluation. Message pass

DOI: 10.24818/18423264/55.1.21.13 
Mihaela Muntean, Claudiu Brândaş, Marian Pompiliu Cristescu, Diana Matiu

\section{CONCLUSION}

SAP CPI is a mature integration platform, open and flexible with different methods for consuming cloud services, open Application Programming Interfaces (APIs) and software developer kits.

The DSR process based on an agile SCRUM demarche describes a framework for successful CIC integration processes. Exemplified on a study case in the SAP environment, the approach is applicable to any CIC integration.

\section{Discussion}

Currently, companies must be able to sustain, monitor and control all kind of business processes that are distributed across different locally installed and/or cloud-based systems. Nowadays, Enterprise Application Integration models in large business environments, including partners, customers and suppliers imply the integration of different Enterprise Resource Planning solutions with other Enterprise systems.

Due to the evolution of cloud computing technologies, the variety of SaaS solutions and other XaaS services, and the advantages of the cloud systems, more and more businesses are moving to the cloud. The widespread business processes imply real-time communication, data accessibility from any location. Therefore, cloud integration approaches are actual research priorities. Any successful implementation enriches the best practices portfolio. With these general considerations, we have introduced three approaches: Cloud-to-Cloud, Cloud-Integrator-Cloud and Cloud-Cloud-Cloud integration. The proposed CIC integration framework in Figure 5 can be applied to any concrete integration project in a similar way as the conducted implementation in the SAP cloud environment.

DSR in information systems has as an objective the creation of an artefact to solve real-life, business problems (Hart, 2010), (Hevner, 2015). According to Hart, the artefact represents a proper solution of a "heretofore unsolved and important business problem".

References on DSR identified in literature (see Hevner, 2004) consist of a combination of general design and development phases, like identification, design, development and testing. Vaishnavi et al. introduced in 2004 a general DSR process model (Figure 2). Other DSR process models are subject of a synthetic analysis in Van der Merwe's paper that is referenced at the end. Each process phase has an established output (Table 2). The symbiotic approach of DSR with SCRUM methodology conducts to an agile integration process (Table $3)$. 
Improving Cloud Integration Using Design Science Research

Table 2. DSR process model

\begin{tabular}{|l|l|}
\hline PHASE & OUTPUT \\
\hline $\begin{array}{l}\text { Awareness of the } \\
\text { problem }\end{array}$ & $\begin{array}{l}\text { Approach proposal: STEP 1 - STEP 5 for CIC } \\
\text { integration }\end{array}$ \\
\hline Suggestion & Tentative design: Integration scenario (Figure 5) \\
\hline Development & $\begin{array}{l}\text { Artefact: Integration framework (Figure 1); } \\
\text { Integration process modeled by SAP CPI (Figure 7) }\end{array}$ \\
\hline Evaluation & $\begin{array}{l}\text { Performance measures: Evaluation of the message pass } \\
\text { (Figure 8) }\end{array}$ \\
\hline Conclusion & $\begin{array}{l}\text { Results: Integration implementation - data transfer from } \\
\text { sender (SAP Hybris Commerce) to receiver (SAP C4C). } \\
\text { Communication of the results }\end{array}$ \\
\hline
\end{tabular}

Table 3. DSR - SCRUM

\begin{tabular}{|c|c|c|}
\hline DSR STEP & SCRUM - ACTIVITY & SCRUM - SPRINT \\
\hline STEP 1. Awareness of the Problem & \multirow{2}{*}{$\begin{array}{l}\text { A0 Awareness of the problem and Tentative } \\
\text { Design }\end{array}$} & \multirow{2}{*}{ Sprint 0} \\
\hline STEP 2. Suggestion & & \\
\hline \multirow{7}{*}{ STEP 3. Development } & A1 Defining the message & \multirow[b]{2}{*}{ Sprint 1} \\
\hline & $\begin{array}{l}\text { A2 Creating the web service between SAP Hybris } \\
\text { Commerce and SAP CPI }\end{array}$ & \\
\hline & $\begin{array}{l}\text { A3 Creating the web service between SAP CPI } \\
\text { and SAP C } 4 \text { C }\end{array}$ & Sprint 2 \\
\hline & A4 Creating the Main Integration Flow & Sprint 3 \\
\hline & A5 Creating the Security Material Artefact & \multirow{4}{*}{ Sprint 4} \\
\hline & A6 Creating the Error Handling Sub-process & \\
\hline & A7 Creating the Value Mapping Artefact & \\
\hline \multicolumn{2}{|l|}{ STEP 4. Evaluation* } & \\
\hline STEP 5. Conclusion & A8 Communicating the result & Sprint 4 \\
\hline
\end{tabular}

*evaluation after each sprint and a final evaluation

DOI: $10.24818 / 18423264 / 55.1 .21 .13$ 
Mihaela Muntean, Claudiu Brândaş, Marian Pompiliu Cristescu, Diana Matiu

Design science research is a problem-solving methodology for developing socio-technical artefacts. The DSR process steps facilitate the integration in the cloud for any CIC integration problem:

- Awareness of the problem: the identified problem is refined. A CIC integration of two SAP Cloud software will be of interest to more than one entity (company).

- Suggestion: the "artefact design" is proposed. Starting from a specific problem (integration of SAP Hybris Commerce with SAP C4C) the integration approach is generalized in order to be relevant to more entities.

- Development: a practice-based design grounds the development of the integration problem. The SCRUM paradigm brings innovation to the artefact, in fact the integration framework and further the integration process.

- Evaluation: a rigorous evaluation of the artefact is essential, e.g. completeness, consistency, accuracy.

- Conclusion: the problem and its importance, the artefact, its utility and novelty, the rigor of its design and its effectiveness are subject of the communication.

\section{Conclusion}

Integration projects of cloud applications manage to streamline the business processes in organizations. The involved systems, SaaS services are integrated. Data introduced in one system is used in the other one.

Integration approaches are based on best practices in the field. Beyond these, research initiatives are necessary to improve the integration framework and to establish general applicable principles. Design Science Research is used in IT/information system projects to solve real-time problems. DSR is an iterative methodology consisting of five steps: awareness of the problem, suggestion, development, evaluation and conclusion. Design science theories and research practices bring improvement to the integration context.

SCRUM methodology, an agile project management methodology, delivers the outcomes "after the most valuable work has been completed" by implying all stakeholders. The DSR-SCRUM framework proposed for CIC integration can be extrapolated, with adaption to the context, to software projects in general.

\section{REFERENCES}

[1] Chody, J. (2018). IaaS, PaaS and SaaS: The Cloud Comparison Guide, [available at:

https://community.hpe.com/t5/Transforming-IT/IaaS-PaaS-and-SaaS-The-CloudComparison-Guide-2018-Update/ba-p/7017543\#.Xd112ugzbIV]; 
Improving Cloud Integration Using Design Science Research

[2] Columbus, L. (2018), State of Enterprise Cloud Computing, [available at: https://www.forbes.com/sites/louiscolumbus/2018/08/30/state-of-enterprise-cloudcomputing-2018/];

[3] Doug, M. F. (2019), What is XaaS, [available at: https://stonebridgeinnovations.com/what-is-xaas/];

[4] Gil, D., Johnsson, M., Mora, H., Szymanski, J. (2019), Review of the Complexity of Managing Big Data of the Internet of Things; Complexity Special Issue on Advances in Architectures, Big Data, and Machine Learning Techniques for Complex Internet of Things Systems, https://doi.org/10.1155/2019/4592902;

[5] Owen, C. (1997), Understanding Design Research. Toward an Achievement of Balance; Journal of the Japanese Society for the Science of Design 5(2): 3645 ;

[6] Pillai, K. (2015), SAP C4C and Hybris Commerce (Integration B2C Use Cases), available at: https://sapcrm7.wordpress.com/2015/11/01/sap-c4c-andhybris-commerce-integration-use-cases/;

[7] Sharma, P. (2018), SAP C4C and SAP Commerce - Integration Highlights, available at: https://blogs.sap.com/2018/12/02/sap-c4c-and-sapcommerce-integration-highlights/;

[8] Beck, R., Weber, S. Gregory, R.W. (2013), Theory-Generating Design Science Research; Hawaii International Conference on System Sciences (HICSS), available at: https://www.researchgate.net/publication/251237390_TheoryGenerating_Design_Science_Research;

[9] Linthicum, D.S. (1999), Enterprise Application Integration; Addison-Wesley Professional: Boston, MA, USA, ISBN 0201615835;

[10] Hart, D.N., S. D. Gregor, S.D. - editors. (2010), Information Systems Foundation: The Role of Design Science, E - Press, ISBN: 9781921666353 (eBook);

[11] Hevner, A.R., S. Chatterjee, S. (2015), Design Science Research in

Information Systems, in: Association for Information Systems. Reference Syllabi, ed.: J. vom Brocke, Eduglopedia.org, available at:

http://eduglopedia.org/reference-yllabus/AIS_Reference_Syllabus_

Design_Science_Research_in_IS.pdf;

[12] Hevner, A.R. et. all (2014), Design Science in Information Systems Research, MIS Quarterly, vol. 28, No. 1;

[13] Kapinga, A.F., Montero, C.S., Mbise, E.R. (2019), Mobile Marketing Application for Entrepreneurship Development: Code sign with Women

Entrepreneurs in Iringa, Tanzania, available at: https://onlinelibrary.wiley.com/doi/full/10.1002/isd2.12073;

[14] Kolluru, N.V.S., N. Mantha, N. (2013), Cloud Integration - Strategy to Connect Applications to Cloud; Annual IEEE India Conference (INDICON), available at: https://ieeexplore.ieee.org/document/6726096;

DOI: 10.24818/18423264/55.1.21.13 
Mihaela Muntean, Claudiu Brândaş, Marian Pompiliu Cristescu, Diana Matiu

[15] Leoz, G., S. Petter, S. (2018), Considering the Social Impacts of Artefacts in Information Systems Design Science Research; European Journal of Information Systems 27(2): 154170;

[16] March, S.T., G. Smith, G. (1995), Design and Natural Science Research on Information Technology; Decision Support Systems, 15(4):251266;

[17] Mittal, S., Negi, N., Chauhan, R. (2017), Integration of Edge Computing with Cloud Computing; International Conference on Emerging Trends in Computing and Communication Technologies (ICETCCT), Publisher: IEEE,1-6; [18] Muntean, M., Brandas, C., Cirstea, T. (2019), Framework for a Symmetric Integration Approach; Symmetry, 11(2), 2019, available at: https://www.mdpi.com/2073-8994/11/2/224;

[19] Takeda, H., Veerkamp, P., Tomiyama, T., Yoshikawam, H. (1990), Modelling Design Processes. AI Magazine Winter: 37-48;

[20] Palos-Sanches, P. R. (2017), Cloud Computing (SaaS) Adoption as a Strategic Technology: Results of an Empirical Study; Mobile Information Systems, https://doi.org/10.1155/2017/2536040;

[21] Uldall-Espersen, T., Frøkjær, E., Blanford, A. (2007), Increasing the Impact of Usability Work in Software Development; Proceedings of the Conference on Human Factor in Computing Systems (CHI2007), San Hose, California, USA, available at:

https://www.researchgate.net/publication/221514531 Increasing the impact of us ability_work_in_software_development;

[22] Van der Merwe, A., Gerber, A., Schmuts, H. (2017), Guidelines for Conducting Design Science Research in Information Systems, available at: http://www.altavandermerwe.com/resources/Pre print\%20Draft.pdf;

[23] Vaishnavi, V., Kuechler, B., Petter, S. (2019), Design Science Research in Information Systems, available at: http://desrist.org/desrist/content/designscience-research-in-information-systems.pdf;

[24] Vaishnavi, V., B. Kuechler, B., S. Petter, S. (2004), Design Science Research in Information Systems, (created in 2004 and updated until 2015 by Vaishnavi, V. and Kuechler, W.); last updated (by Vaishnavi, V. and Petter, S.), June 30, 2019. URL: http://www.desrist.org/design-research-in- informationsystems/;

[25] Wang, S.H., Chen, D.A. (2014), Cloud Framework for Enterprise

Application Integration on SOA; Proceedings of the International Conference on Software Intelligence Technologies and Applications \& International Conference of the International Conference on Software Intelligence Technologies and Applications \& International Conference on Frontiers of Internet of Things, Hsinchu, Taiwan. 